\title{
Educación sexual, eugenesia y moral en el pensamiento de Paulina Luisi. La experiencia de la cátedra de Higiene Social (Uruguay, 1926-1930)
}

Fernanda Sosa Cedrani

CFE-ANEP (Uruguay)

totifernanda@gmail.com

\section{( Santiago Zemaitis \\ UNLP/UNQ (Argentina) \\ zemaitis.santiago@gmail.com}

Fecha de recepción 17/2/2020 Fecha de aceptación 5/10/2020

\begin{abstract}
Resumen
El objeto de este artículo es el abordaje de la concepción de educación sexual desarrollada por la primera médica uruguaya Paulina Luisi, una de las pioneras en el Río de la Plata en asumir la relevancia y pautar ciertos principios en torno a la enseñanza sexual. Para ello, nos interesa centrarnos en su cátedra de Higiene Social (1926-1930) destinada a estudiantes de las escuelas normales, experiencia curricular que se constituyó en uno de los primeros intentos por institucionalizar la educación sexual en la formación docente normalista. Indagaremos cómo la sexualidad y sus problemáticas se expresaron en el discurso pedagógico de Luisi recorriendo los fundamentos morales, pedagógicos y científicos que sustentaron su propuesta. El artículo, inscripto en el campo de la Historia de la Educación, particularmente en la Historia de la educación sexual, avanza con un análisis cualitativo de diversas fuentes como "Algunas ideas sobre Eugenia" (1916), Plany métodos de Enseñanza Sexual (1919), "Para una mejor descendencia" (1919), Ensayo sobre Enseñanza biológico-eugenésica (1921, 1923), Una Cátedra de Higiene Social (1950). Bajo las lecturas de estos escritos, pretendemos visibilizar los fundamentos teóricos de las normas morales que Luisi ensayaba para definir la sexualidad y los medios más adecuados para la formación en dicha materia, en articulación con las ideas eugenésicas que tiñeron buena parte del pensamiento médico y social en los inicios del siglo XX. La educación sexual que proponía Luisi tuvo como objetivo el "control de los instintos" y la formación de una "inteligencia informada y responsable" para con la descendencia (responsabilidad genésica); una educación sexual articulada también con la prevención del contagio de las enfermedades venéreas y con la generación de descendencia sana que permitiera
\end{abstract}


el mejoramiento de la raza. En su proyección, imaginó una educación sexual en todos los niveles de la enseñanza en la educación pública, proponiendo un papel relevante a las familias y a los docentes. Cuestionó las posturas que optaban por el silenciamiento de los temas vinculados a la sexualidad a niños y jóvenes y, como se hará notar en su experiencia de cátedra, su apuesta curricular y metodológica pretendió colocar en el debate pedagógico-político a la sexualidad y sus problemáticas, un ensayo pedagógico que enlazó ciencia, moral y eugenesia. ${ }^{1}$

Palabras clave: Paulina Luisi; educación sexual; Eugenesia.

\begin{abstract}
The object of this article is the conception of sexual education developed by the first Uruguayan doctor Paulina Luisi, one of the pioneers in the Río de La Plata to assume relevance and guide certain principles around sexual education. To do this, we are interested in focusing on his Chair of Social Hygiene (1926-1930) for students of the Normal schools, a curricular experience that meant being one of the first attempts to institutionalize sex education in normalist teacher education. We will investigate how sexuality and its problems were expressed in Luisi's pedagogical discourse, covering the moral, pedagogical and scientific foundations that supported his proposal. The article, inscribed in the field of the History of Education, particularly in the History of sex education, advances with a qualitative analysis from various sources such as "Some Ideas on Eugenia" (1916), Plan and Methods of Sexual Teaching (1919), "A Better Offspring” (1919), Essay on Biological-Eugenic Teaching (1921, 1923), A Chair in Social Hygiene (1950). Under the readings of these writings, we intend to make visible the theoretical foundations of the moral norms that Luisi tried to define sexuality and the most appropriate means for its formation, in articulation with the eugenic ideas that stained much of medical and social thought at the beginning of the 2oth century. The sexual education proposed by Luisi had as its objective the "control of the instincts" and the formation of an "informed and responsible intelligence" towards the offspring (genetic responsibility); a sexual education also articulated to the prevention of the venereal diseases contagion and in the generation of healthy descendants that would allow the improvement of the race. In his projection, he imagined sexual education at all levels of education in public education, proposing a relevant role to families and teachers. He questioned the positions that opted for the silencing of issues related to sexuality to children and youth, and. As will be noted in his Chair experience, his curricular and methodological commitment sought to place sexuality and its problems in the pedagogical-political debate, a pedagogical essay that linked science, morals and eugenics.
\end{abstract}

Key Words: Paulina Luisi; sex education; eugenics.

\title{
Introducción. Aportes y posiciones de Paulina Luisi
}

Paulina Luisi fue una figura central en la historia uruguaya moderna por varias razones. Habiéndose formado inicialmente como maestra en el nivel elemental y superior, logró ser la primera mujer en Uruguay en obtener el título de Doctora en Medicina en 1908. Tuvo también una gran injerencia en los asuntos sociales y políticos, en especial en su lucha a favor de los derechos políticos de las mujeres. Lideró en 1916 la fundación del Consejo Nacional de Mujeres - que funcionó en su propia casa-y fue, asimismo, cofundadora del Partido Socialista del Uruguay. Reconocida por su lucha en el campo de los derechos políticos y civiles de las mujeres, fue una

1 Queremos expresar nuestro agradecimiento al Dr. Pablo Scharagrodsky por su detenida y siempre cuidadosa lectura. También agradecemos los aportes realizados por el comité evaluador del artículo. 
de las principales defensoras del sufragio femenino. Sus ideas y su actividad pública la posicionaron en los debates de la época en el contexto internacional. Así, llegó a representar a su país en la Comisión Consultiva contra la Trata de Mujeres y Niños de la Sociedad de las Naciones y formó parte del Comité de Técnicos contra la Trata en Ginebra. En el campo de la medicina, se especializó en el área de Ginecología y en la salud de mujeres y niños.

Luisi participó en los debates educativos en el Uruguay de inicios del siglo XX y se manifestó a favor del acceso de las mujeres a la educación secundaria, terciaria y al ejercicio de distintas profesiones. Su postura es valorada por su lucha por derechos civiles y políticos para las mujeres y la apertura de ámbitos para el estudio y el trabajo femeninos. Asimismo, defendió la modalidad coeducativa como formato pedagógico. Sin embargo, apoyó la creación de una institución de enseñanza secundaria exclusivamente femenina como manera de ampliar posibilidades de acceso a la educación a mujeres provenientes de familias con prejuicios frente a la coeducación de los sexos (Sosa, 2018).

El presente trabajo, enmarcado disciplinariamente en la historia de la educación y en la particular historia del campo de la educación sexual, indaga respecto a la forma en que Luisi ha promovido, estudiado y difundido - tanto en su medio local como también en los debates médicos y políticos internacionales-aproximaciones en torno a la sexualidad y sus problemáticas sociales vinculadas (el onanismo, el problema del instinto sexual, la prostitución, la trata sexual, el mejoramiento de la "raza"), como también sus líneas más propositivas en materia de "educación sexual" e "instrucción sexual".

El objeto de este artículo es la educación sexual dentro de la cátedra de Higiene Social para estudiantes de las escuelas normales dictada por Paulina Luisi (1926-1930). Se indaga la forma en que se expresa la sexualidad en el discurso pedagógico de Luisi y en los fundamentos que sustentaron este discurso. Para realizar este cometido, en los primeros apartados se presentan las propuestas de educación sexual realizadas por Paulina Luisi en Plany métodos de Enseñanza Sexual (1919) y en el Ensayo sobre Enseñanza biológico-eugenésica $(1921,1923)$. Entre las disputas de sentido de la educación sexual de principios del siglo XX en el Uruguay, Luisi argumentó a favor de la educación sexual en todos los grados de enseñanza, enfrentó las posturas que optaban por el silenciamiento de los temas vinculados a la sexualidad, hizo propuestas curriculares y metodológicas, entendió que la sexualidad no era un ámbito exclusivo de ciertas profesiones y concedió un papel relevante a las familias y a los docentes. En las definiciones de enseñanza sexual y en los contenidos se enlazan ciencia, moral y eugenesia.

En el segundo apartado dedicado a la cátedra de Higiene Social para estudiantes normalistas, se caracteriza su organización, metodología, definiciones y contenidos. Esta iniciativa fue muy relevante porque es la experiencia por la cual se concreta la educación sexual en términos institucionales, ya que la Ley que crea la cátedra es la única aprobada entre las leyes con propuestas de educación sexual que hubo en la década de 1920. Además, la incorporación de contenidos eugenésicos en la currícula de la cátedra significa un espacio de concreción de las ideas eugenésicas. Para todo ello se toman escritos publicados, discursos pronunciados y su experiencia pedagógica desde la cátedra de Higiene Social en las Escuelas Normales de Montevideo (19261930), cátedra que se complementaba con actividades en el Museo Pedagógico con amplia concurrencia de público de diferentes perfiles.

Por último, se analiza la postura eugenésica de Luisi - que acordaba con procedimientos de la eugenesia positiva y negativa-, se vinculan las ideas eugenésicas con las propuestas de educación sexual y la preferencia por la eugenesia de Pinard. 
En este apartado se presentan fuentes clave y se dialoga con indagaciones en torno a la eugenesia. Las fuentes aludidas son los folletos producidos por Luisi "Algunas ideas sobre Eugenia" (1916) y "Para una mejor descendencia" (1919) y un manuscrito que se presume parte de los contenidos de la cátedra.

Como ya han aportado varios estudios, la educación sexual, entendida como control de los instintos y como estrategia profiláctica para evitar los "males venéreos", ha sido en la región latina una herramienta privilegiada de los discursos e iniciativas del movimiento eugenista (Miranda, 2005, 2011; Di Liscia, 2008; Biernat, 2007; Palma y Palma, 2007; Queirolo, 2013; Miranda y Vallejo, 2014).

En términos disciplinares, pretendemos realizar un aporte a la reconstrucción de las diferentes tradiciones, historias locales y agentes sociales que marcaron tendencias pioneras, promovieron prácticas pedagógicas y abrieron acalorados debates sociales en torno a la educación sexual, en especial a aquellos trabajos que han centrado su atención en figuras paradigmáticas entre finales del siglo XIX y la primera mitad del siglo XX en la región del Cono Sur de nuestro continente (Nari, 2004; Lavrin, 2005; Miranda y Vallejo, 2005; Darré, 2005; Miranda, 2011; Southwell, 2011; Jones, Figari y Barrón López, 2012; Scharagrodsky y Zemaitis, 2020). En definitiva, nos interesa contribuir en la reconstrucción de discursos, posiciones e iniciativas en la historia de la educación sexual, un objeto de estudio que muy recientemente se comienza a explorar y que hasta ahora ha tenido menciones laterales en investigaciones mayores (Zemaitis, 2016). En esta línea, sostendremos que explorar el discurso pedagógico² de Luisi permite reconocer las improntas (científicas, políticas y pedagógicas) típicamente modernas de cómo se ha concebido la formación de la sexualidad, sus finalidades, sus riesgos y sus posibilidades de producir una transformación social en torno a determinadas problemáticas sanitarias y educativas.

\section{La "enseñanza sexual"}

En el Uruguay de inicios del siglo XX hubo proyectos e iniciativas de educación sexual que no lograron concretarse en las instituciones educativas a excepción de la cátedra de Higiene Social en las Escuelas Normales de Montevideo. En 1922, el diputado uruguayo Mateo Legnani presentó un proyecto de ley sobre educación sexual que no fue aprobado, y en 1924, el senador Alejandro Gallinal presentó un nuevo proyecto que resultó parcialmente aprobado pero no en lo referido a educación sexual (Darré, 2005). Esta autora expresa que, en los albores del siglo pasado en el entorno montevideano, los debates sobre educación sexual rondaron en torno a tres asuntos: primero, sobre el acuerdo general sobre la peligrosidad de los instintos sexuales, se discutió si la educación sexual contribuía a despertarlos o a acallarlos; segundo, en cuanto a quién contaba con la competencia profesional para intervenir en este saber; y tercero, si se trataba de un asunto individual o colectivo. Estos interrogantes se verifican en un Uruguay en el que crecían los efectos de la secularización, la influencia de la Iglesia decrecía y aumentaba la influencia del saber y poder médicos. Los proyectos mencionados no lograron consolidar la educación sexual dentro de las instituciones educativas, aspecto que Darré analiza como "una de las primeras rupturas en el proyecto moderno" (Darré, 2005: 59). Para esta autora, esto ocurría porque todos los actores que debatieron tenían posturas similares en torno a la peligrosidad de los instintos y eso hizo que las posturas más progresistas se afiliaran

2 Adscribimos a la definición que propone Silvana Darré de discurso pedagógico, categoría teórica de raigambre foucaultiana que "reúne lo que es seleccionado del conjunto de los discursos sociales para su circulación en términos pedagógicos. La especificidad del discurso pedagógico radica en su posibilidad de vehiculizar otros discursos sociales, políticos, jurídicos, médicos" (2008: 201). 
a tradiciones pedagógicas más autoritarias (ibídem). Incluso, dos décadas antes, en el inicio del novecientos, encontramos propuestas educativas informales, sobre todo alrededor de la sífilis y la tuberculosis, como unas de las mayores preocupaciones de los médicos higienistas. Aunque no eran pensadas como "educación sexual" (al menos no articuladas a este sintagma), se trató de iniciativas que pretendían infringir terror en los jóvenes varones en relación a las prácticas sexuales, como el folleto "Prevenir a los jóvenes", difundida en 1906 por el presidente del Consejo Nacional de Higiene, Alfredo Vidal y Fuentes, o bien la "Cartilla sobre la Tuberculosis y Nociones de Higiene Preventiva" de 1909, escrita por el médico Sebastián B. Rodríguez, destinada a ser difundida en las escuelas uruguayas. Este tipo de producciones de divulgación, por el tono de sus discursos, contenían el terror como método educativo y profiláctico (Díaz Berenguer, 2012). Como veremos, la proyección pedagógica y social de Luisi pretendía ir más allá de este tipo de campañas articulando una propuesta de educación sexual que incluyera estos y otros aspectos, no solo centrada en enfermedades venéreas puntuales ni en una propuesta únicamente médica.

En este contexto, Luisi presentó su Plan y Métodos de Enseñanza Sexual en 1919, el mismo que ya había presentado en 1906 a las autoridades de la Dirección de Instrucción Pública. Se trató de una propuesta para incorporar "nociones de enseñanza sexual" en los programas de las escuelas normales y de tercer grado. ${ }^{3}$ La respuesta que recibió en ese entonces fue que la llamaran "revolucionaria" y "anarquista". Luisi insistió con la presentación de un plan con métodos en el que definía: quién debía estar a cargo de la "enseñanza sexual", cuándo debía comenzar, dónde, qué temas abarcaría y cómo debía darse (Luisi, 1922: 217-223).

Con estos interrogantes, formulados y ensayados a lo largo de sus escritos, artículos y comentarios públicos en torno a la "enseñanza sexual", Luisi marcó objetivos pedagógicos y sociales clave que serían luego premisas de varias campañas e iniciativas sobre las relaciones entre la educación y la sexualidad durante la primera parte del siglo XX. En aquel período, la sexualidad (sus prácticas, legitimidades, expectativas políticas y sociales) se encontraba unida a la reproducción como finalidad, al control sobre el instinto sexual en los niños y adolescentes y a una perspectiva profiláctica y eugénica sobre la reproducción. Introdujo de forma temprana también la noción de responsabilidad, término que tendría persistencia en algunos discursos provenientes del amplio y heterogéneo campo de debate de la educación sexual.

De este modo, la propuesta de Luisi disputaba sentidos sobre la enseñanza de la sexualidad en relación al mutismo sobre este tema; se oponía a conjeturar que la educación e instrucción sexual se trataban de aspectos de la formación del carácter en su sentido individual, reducido a la educación familiar. Por el contrario, defendía que aquellos temas debían estar en el marco de la enseñanza pública y que debían ser legislables, como asuntos propios del sistema educativo y de los responsables del mismo. Una tesis similar sostuvo su amiga y colega argentina Raquel Camaña, quien exhortaba para que la educación sexual fuera un derecho efectivizado por el Estado, y no un "asunto delicado" a ser tratado por el derecho de los padres (Camaña, 1910, 1912, 1916).

3 "Escuelas normales" es el nombre que recibían por uso los Institutos Normales para la formación magisterial. En 1906, año en que Paulina Luisi propuso la incorporación de nociones de enseñanza sexual en los programas de estudio, había dos Institutos Normales, el de Señoritas y el de Varones, ambos en la ciudad de Montevideo. Por otro lado, las "escuelas comunes" eran de tres grados, según los años de estudio que contuvieran: 1er grado de $1^{\circ}$ a $4^{\circ}, 2$ do grado de $5^{\circ}$ a $6^{\circ}$ y 3 er grado de $7^{\circ}$ a $8^{\circ}$ (Palomeque, 2012: 414). Según este autor, refiriéndose a la década del 30 del siglo XX: "En el país existen solo dos escuelas de zer grado". La propuesta inicial de Paulina Luisi era entonces incorporar algunas nociones de enseñanza sexual a estudiantes de magisterio y en los años superiores de las escuelas comunes. Cuando en 1919 retomó la idea de proponer un "Plan y Métodos de Enseñanza Sexual" abarcó todos los niveles educativos. 
En la propuesta pedagógica de Luisi la "enseñanza sexual" se encontraba organizada, primordialmente, en tres grandes núcleos temáticos:

I Una parte relativa a la educación propiamente dicha, esto es, al sometimiento y dirección del instinto y sentimiento sexuales a las normas establecidas por la moral individual y sociales; en otros términos el sometimiento del instinto sexual al dominio de la voluntad reflexiva, advertida y consciente, por obra de la educación de esta facultad anímica y la formación del carácter (Educación Moral).

II Una segunda parte relativa a la instrucción, esto es, a los conocimientos científicos atingentes a los fenómenos de la reproducción de la especie: historia natural anatomía y fisiología humanas, higiene y profilaxis (Instrucción Científica).

III Una tercera parte que comprende el conocimiento de la deontología social en la parte relativa a las cuestiones de la generación (Instrucción Eugénica) (Luisi, 1950: 82).

En efecto, su planteo no sostenía una mirada reduccionista a la dimensión biológica o anatómica: "La enseñanza sexual es mucho más vasta, más trascendente, que el simple conocimiento de determinadas funciones que cumple o no cumple el organismo, o que el conocimiento de nociones de profilaxia y de higiene" (Luisi, 1922: 205). En esta enseñanza sexual, Luisi distinguía la "instrucción sexual" de la "educación sexual". La primera se componía de los conocimientos científicos de la materia, mientras que el segundo término "encierra en sus lecciones el evangelio de una nueva moral, basada en el respeto humano y en la responsabilidad individual dentro de la vida colectiva" (ibídem: 206).

Así, las bases pedagógicas y epistemológicas para su proyecto de educación sexual quedaron circunscritas a la moral, la ciencia y la eugenesia. Esta definición tuvo una buena aceptación local, incluso, llegó a proyectarse y logró obtener legitimidad en el debate internacional de ese tiempo. Presentada en Uruguay en 1921 ante el Consejo Médico Nacional, esta definición viajó a congresos y reuniones internacionales de expertos y fue logrando aceptación y reconocimiento. Su divulgación se dio desde el Congreso de Higiene Social en 1923 con sede en París, donde tuvo un significativo apoyo y aprobación, hasta el Tercer Congreso Italiano contra la Trata de Mujeres y Niños, celebrado ese mismo año en Milán. En este evento se aprobó y adoptó esa definición tripartita que posteriormente fue publicada en diversos medios internacionales de gran relevancia hasta $1936 .{ }^{4}$ Originalmente escrita en francés, se tradujo luego al español, posibilitando su difusión en los discursos de las entidades y ligas argentinas de profilaxis y eugenesia, a través de folletos de circulación también entre los países del Cono Sur. ${ }^{5}$

Para Luisi la enseñanza sexual debía comenzar “desde que el niño frecuenta la escuela y aprovechando las ocasiones oportunas para ello" (ibídem: 218). Debía realizarse en todas partes, desde las escuelas normales, las de adultos, las primarias, secundarias, preparatorias, en las escuelas hogares y hasta los niveles superiores de formación como las facultades (ibídem: 218-219). Acerca de cuándo se debía iniciar la educación sexual, señaló:

4 Algunos que menciona la propia Luisi fueron la Presse Médicale (París, 1923), "Educación sexual" (Lisboa, s/f), Actas del Congreso Médico Nacional (Montevideo, 1921) y el Bulletin des Groupes Feministes de l'Enseignement Laique (París, 1925).

5 Según Luisi, su traducción y circulación amplia en el Río de la Plata fue posible gracias a la traducción realizada por la Liga Argentina de Profilaxis Social en 1936. También se imprimió en folletos de la Asociación Argentina de Eugenésica [sic]. 
Cuestión esta sobre la que no se han puesto aún de acuerdo los pedagogos, higienistas y cuantos se han ocupado de este asunto. Los más timoratos estiman que es del resorte de la escuela secundaria, otros, que se debe reservar para el último año de la primaria. Una ínfima minoría (...) estima que debe comenzarse desde los cuatro o cinco años; yo, afirmo que no hay más límite de edad que aquel en que las oportunidades de hablar de ello se presentan. (ibídem)

Sin marcar límites etarios, se alejó de posturas "timoratas" y defendió más bien una posición disruptiva. También generó novedades con su propuesta acerca de quién debía encargarse de la enseñanza sexual. Los responsables de su desarrollo debían ser tanto los padres como los maestros. Puntualmente, consideraba que los "padres deben ser preparados por los maestros al cumplimiento de sus deberes de educadores primarios" (ibídem: 217). Es decir, las maestras y los maestros en la educación sexual debían dirigirse tanto al alumnado como a las familias.

De este modo, se aprecia una clara intención pedagógica en estos escritos por la manera en que imaginaba el desarrollo de un tipo de educación sexual dentro y fuera del ámbito formal del sistema educativo. Por otro lado, no sostuvo una mirada restringida del saber sobre la sexualidad y su enseñanza, es decir no la colocó como un ámbito exclusivo de ciertas profesiones o campos científicos en manos de "expertos".

Acerca del método pedagógico para la enseñanza sexual, Luisi creía que mientras en las escuelas secundarias de adultos, las normales y las facultades debían establecerse programas específicos de enseñanza sexual que permitieran un "estudio racional de los conocimientos que requiere y con la extensión que a cada uno corresponde" (ibídem: 221), en las escuelas primarias la enseñanza sexual debía estar "diluida" en las asignaturas que ya existían en los programas (ibídem: 222).

En cuanto a la organización curricular, los contenidos del Plan y métodos de enseñanza sexual -agrupados en cuatro asignaturas - abordaban, en la asignatura Higiene, la profilaxis de las enfermedades venéreas y la "profilaxia e higiene de las perversiones sexuales (masturbación, etc.)" (ibídem: 220). Todo esto era lo relativo a la instrucción sexual. Luisi definió la higiene social como la "rama de la profilaxis que se ocupa de contrarrestar las enfermedades de la colectividad con la higiene colectiva (...) Se trata pues de prevenir las enfermedades sociales como con la higiene privada se pretende prevenir las enfermedades individuales" (Luisi, 1925: 5). Las enfermedades sociales son las que "aumentan su poder mortífero por las condiciones sociales" (ibídem).

Agregó también contenidos relativos a la "educación sexual propiamente dicha" que era "La castidad, como medio de dar mayor vigor al cuerpo" (Luisi, 1922: 220), la necesidad de hacer primar a la voluntad por encima de los deseos, la castidad, la coquetería e "incitaciones inconscientes de la mujer en la búsqueda de marido". También se hacían visibles como contenidos curriculares la seducción y las responsabilidades frente a la familia y los hijos, ya que la maternidad era definida por Luisi como la "más alta función de la especie" (ibídem). Incorporó también como contenidos sus críticas a la prostitución y a las "lecturas libertinas, frecuentación de cabarets y espectáculos licenciosos, de las lecturas y conversaciones pornográficas" y la lucha contra la pornografía (ibídem: 219-221).

\section{Educación moral y control del instinto sexual}

Como se puede observar, sexualidad y moral, así como la responsabilidad individual de los propios actos ligados a los problemas colectivos, eran una suerte de equivalencias 
muy propias del pensamiento médico, social y político en los inicios del siglo XX. Se trata de significaciones asociadas que quedaban condensadas en esta propuesta pedagógica. Como señala José Pedro Barrán, el "culto a la salud" como un valor absoluto "implicó un cambio en la titularidad de los dirigentes y creadores de las conductas morales aceptadas, pues el cura fue sustituido por el médico en la dirección de las conciencias individuales" (Barrán, 1992: 11).

Aún queda por comprender cómo justificaba Luisi estas normas de conducta para la sexualidad, punto que se aclara en su Ensayo sobre Enseñanza biológico-eugenésica (Doctrina-Metodología) ${ }^{6}$ de 1921. Parte de la fundamentación de este Ensayo versaba sobre "La moral biológica como fundamento de la moral práctica". Conviene detenerse en estos conceptos porque marcan cómo desde el saber médico se justificaba la incursión en la recomendación de normas de conducta a las poblaciones. Luisi partía de la idea de que "el instinto sexual se ha desviado de su función natural que es la reproducción de la especie" (Luisi, 1950b: 36). Esto ha generado en el campo de la Higiene una preocupación enorme por la difusión de las enfermedades venéreas y en la moral por "la disolución y el desorden de las costumbres actuales" (Luisi, 1950a: 36). Según Luisi, la preocupación por la higiene y la moral eran "la necesidad de la salud física y psíquica" y en defensa de la salud es que se dictaminaban principios de orden moral. Expresaba que "La moral debe fundarse en la ciencia" (Luisi, 1950b: 37). Nuevamente sexualidad, moral y reproducción quedaban fuertemente asociadas en su propuesta.

\section{Educar el instinto}

En estas reflexiones pedagógicas (más que médicas), Luisi volvía sobre los tres grandes ámbitos o dimensiones de la acción pedagógica para la enseñanza sexual: educación propiamente dicha o educación moral, instrucción científica e instrucción eugenésica. En el Ensayo, Luisi desarrolló la siguiente definición: "La enseñanza sexual es la acción pedagógica que pretende someter el instinto sexual a la acción de la voluntad bajo el contralor de la inteligencia instruida, consciente y responsable" (ibídem: 82).

¿Cómo desarrollar una voluntad que pudiera controlar el instinto sexual? Luisi creía que esto no podía hacerse solamente brindando información, dadas las características del deseo sexual: "Normalmente, el deseo sexual es un impulso capaz de sacrificar a su satisfacción pura, hasta el instinto de la propia conservación. Por eso se ha dicho siempre que el deseo es ciego. Esta locura del amor instintivo es la que debe ser sometida al freno de la voluntad" (ibídem: 88).

Para lograrlo, sugería un entrenamiento como el que se realizaba para incorporar cierto tipo de hábitos como, por ejemplo, los de la higiene corporal. Expresaba que "combatiendo las golosinas, la glotonería, la pereza, soportando el frío y el calor, sufriendo en silencio pequeños dolores, se va desarrollando la resistencia a sensaciones desagradables" (ibídem: 88-89). Agregando a estos esfuerzos físicos otros morales (como combatir el miedo, la cobardía, la mentira, etc.) se "convertirá al niño en un hombre capaz de resistir a la sugestión de los apetitos y las tentaciones" (ibídem: 89).

Vale destacar que Luisi no investía a la infancia con los mantos modernos de inocencia, sumisión y docilidad. Por el contrario, a través de sus escritos y experiencias podemos leer que había reconocido que tanto niños como jóvenes tenían un cierto acceso al saber sobre la sexualidad, ya desde el momento en que los pequeños tenían representación gráfica de las "partes vedadas", tal como admitía en el capítulo IV "Prejuicios-Placer-Pornografía" de su Pedagogía:

6 En este pronunciamiento Luisi reafirmó su postura acerca de que la enseñanza sexual debía darse en todas las edades y en el marco de los programas existentes para que la enseñanza sexual se verificara "sin darse cuenta de ello" (Luisi, 1950b: 79). 


\begin{abstract}
“¿Por qué empeñarse en abrirles los ojos a los niños?”, me decía una señora. “¡Es tan hermoso conservarles la inocencia”! y agregaba enseguida: “¡Para qué ese empeño, si hoy en día ya los tienen demasiado abiertos!”. Está en el convencimiento de todos, que los adolescentes de uno y otro sexo no ignoran esos conocimientos que la familia y la escuela les niegan. Padres y maestros saben perfectamente que los niños están enterados no solo de las elementales nociones sobre la maternidad y la procreación, sino también algo saben, y no siempre como se debe, de las relaciones sexuales. (Luisi, 1950: 51)
\end{abstract}

Frente al silenciamiento de los temas vinculados con la sexualidad, que se imponía por cuestiones morales o por una presunción de inocencia en la infancia, defendió la postura de hacer disponibles los conocimientos y así combatir la ignorancia en estos asuntos.

\title{
La creación de la cátedra de Higiene Social (1926-1930)
}

Las propuestas sobre educación sexual de Paulina Luisi fueron ampliamente difundidas y debatidas, pero no se implementaron dentro del ámbito de todo el sistema educativo como añoraba, sino que se redujeron al ámbito de la formación magisterial. Funcionó así para estudiantes de las escuelas normales una cátedra de Higiene Social, espacio creado por su propia iniciativa y aprobado por ley en febrero de 1925, aunque comenzaría sus actividades curriculares al año siguiente. Esta iniciativa, aunque breve, constituyó el ámbito en el que se consiguió establecer la educación sexual dentro de un programa que era más amplio.

Esta cátedra se extendió hasta 1930, año en que Luisi se jubiló. Sin embargo, la finalización de su dictado tuvo más que ver con "Choques de intereses personales, intrigas, muñequeos" (Luisi, 1950a: 265), por la resistencia que presentaba ante las autoridades de la Escuela Normal o por la tipificación de la cátedra como "inútil" por parte del propio Director del Cuerpo Médico Escolar. Luisi describió la finalización de la cátedra con gran frustración porque creía que se suprimía una tarea que era necesario continuar: "la preparación de los educadores a los problemas sociales y sexuales, y la pedagogía de esta enseñanza” (ibídem: 265).

La cátedra se enmarcó, claramente, dentro del movimiento higienista: varios grupos de médicos y sociólogos veían en el mejoramiento de los programas de salud pública, una forma de mejorar el perfil sanitario urbano bajo varias estrategias, entre ellas la atención preventiva como un elemento clave (Lavrin, 2005). La Higiene era el encumbramiento de la salud y tutelaba la actividad humana en nombre de su mantenimiento (Barrán, 1992: 233). Luisi incorporó un enfoque eugenésico porque "para ella higiene social y eugenesia se correspondían en conceptos y fines" (Espiga, 2015a: 8). En el apartado siguiente se analizan los contenidos de la cátedra y su inscripción en el higienismo y la eugenesia.

La metodología propuesta desde la cátedra contaba con clases teóricas (exposiciones orales, proyecciones luminosas y algunas cintas cinematográficas de la Asociación Americana de Higiene Social), visitas a hospitales y dispensarios (se realizaban en días domingos y festivos y eran facultativas) y lecciones prácticas que se realizaban en escuelas (Luisi, 1950). A estas clases con estudiantes normalistas, se fueron incorporando otras actividades semanales dentro del Museo Pedagógico dirigidas a maestros ya diplomados, pero también abiertas al público en general. Luisi las llamó "Conferencias de Higiene Social" y las incluyó como acciones anexas a la cátedra. Según sus testimonios, "estaban siempre desbordantes de público" (ibídem: 257). 
Pasados los años de aquella iniciativa pionera, Luisi sostuvo que la experiencia de la cátedra, por los estudiantes y sus conferencias anexas, fue de vanguardia y de "cuña combativa":

(...) el hielo ha sido roto, la desventurada Cátedra de Higiene Social realizó, contra viento y marea su rol de vanguardia. Abrió victoriosamente las hostilidades contra los prejuicios y la rutina. (...) Y abierta la cuña combativa, no dudamos que poco a poco se irá completando esta enseñanza para honor de un país que como el nuestro, quiere encontrarse entre las avanzadas del progreso. (Luisi, 1944: 11-12)

En lo que sigue se revisará la organización, los contenidos y las definiciones conceptuales desplegadas en la cátedra, tarea que no ha sido abordada en otras investigaciones sobre los aportes de Luisi y que reviste interés porque esta experiencia fue el ámbito en que se desarrolló pedagógicamente la educación sexual, a pesar de todas las resistencias, ya que fue la única de las propuestas de educación sexual de la época formuladas como proyecto de Ley que consiguió su aprobación. Y, además, porque se constituyó como un medio para la difusión de contenidos de la eugenesia en la currícula magisterial, como también entre otros públicos con las conferencias anexas.

\section{Los contenidos del Programa de la cátedra de Higiene Social}

La cátedra comenzaba su propuesta temática con una definición de las enfermedades sociales y de la Higiene Social. Luego se estudiaban las "enfermedades sociales" y su profilaxis: la tuberculosis, el cáncer, los quistes hidáticos, las enfermedades venéreas, las drogas nocivas, el alcoholismo, las enfermedades mentales. Continuaba el programa con cuestiones de eugenesia? "para entrar por estas nociones a encarar el gran problema de la responsabilidad racial y la profilaxis y la moral de los problemas relativos a la generación" (Luisi, 1950a: 254).

Proseguía luego con tres unidades de su programa a las que llamó "enseñanza sexual". Estos tres núcleos se organizaban de acuerdo con los "deberes en materia de higiene sexual". La primera unidad correspondía a los deberes consigo mismo, la segunda a los deberes para con el otro sexo y la tercera, a los deberes que se derivaban para con el producto de la concepción.

La primera de las unidades contenía temas vinculados a la "represión del instinto". Proponía al respecto la castidad, la continencia, los ejercicios físicos para vencer al instinto sexual (como "frenadores y moralizadores"), la influencia perniciosa de lecturas, imágenes y conversaciones que pudieran incitar al deseo sexual. Agregaba la profilaxis de las enfermedades sexuales y la higiene moral que eran para Luisi la educación de la voluntad para refrenar los instintos (Luisi, 1950a). La defensa de la castidad para ambos sexos ya estaba presente en el Ensayo sobre Enseñanza Biológico-Eugenésica. Allí, Luisi sostenía que resultaba deseable la abstinencia sexual hasta la adultez y buscaba argumentos para contrarrestar la creencia de que los varones no podían reprimir sus deseos sexuales. Sentado esto, se dedicó a criticar a la "pornografía y literatura libertina" porque estas influencias "despiertan un instinto que aún no se sabe dominar" (Luisi, 1950b: 93).

La segunda unidad denotaba el ejercicio de la sexualidad heterosexual entre dos personas. Dejaba espacio para analizar la "seducción e incitación" del hombre y de la mujer y agregaba para la mujer "las modas y los modales provocativos e incitantes". Planteaba luego el concepto de "unidad de la moral" en materia sexual, referido a la contaminación de las enfermedades venéreas y a la fecundación (Luisi, 1950a: 276). Luisi criticaba el doble criterio moral (diferenciado para varones y mujeres) en

7 Luisi expresó que Francis Galton en 1869 había sentado las bases de la ciencia a la que llamó Eugenia en esta publicación (en las siguientes utilizará la palabra eugenesia). 
materia sexual. Mientras para los varones se daban todas las licencias y permisos, se exigía de las mujeres todos los sacrificios. Vale recordar que, tal como lo ha descrito Mario Pecheny, esas licencias tuvieron que ver con la instauración de un canon de una severa moral sexual que ordenó y disciplinó en aquellos años de forma diferente y desigual a hombre y mujeres; todo ello a los fines de asegurar la reproducción de la especie, antes que el placer y el deseo (Pecheny, 2009).

Creía Luisi además, que de esta doble moral surgía la prostitución, "vergüenza de la humanidad" (Luisi, 1950b: 107). Particularmente, cuestionó que se considerara a la procreación como asunto femenino exclusivamente "Es necesario enseñar a los jóvenes de ambos sexos que, dada la finalidad biológica del acto sexual, su realización acarrea los mismos deberes y las mismas responsabilidades para los dos actores. (...) Una sola moral se impone para los dos sexos" (ibídem: 107).

Este último tema fue desarrollado en la tercera unidad: "Enseñanza sexual". Allí Luisi confiaba en que la educación sexual crearía conciencia sobre la responsabilidad paternal con la finalidad de evitar abandonos y cuidar la salud para evitar la transmisión de enfermedades hereditarias, ideas que sostenía a partir de su adhesión a los planteos eugenésicos, como se plantea en el siguiente apartado.

\section{"Para una mejor descendencia": eugenesia e higiene social}

Diego Armus señala que una de las discusiones en los estudios sobre eugenesia en Buenos Aires refiere a su mayor o menor materialización en iniciativas y políticas e ilustra su convicción de que "muchas veces se trata solo de discursos sin tangibles influencias en la reproducción de la vida diaria de la población" (Armus, 2016: 156) con algunos ejemplos: la eugenesia no se incorporó en la currícula de las escuelas normales a pesar de que hubo varios intentos, el fichaje biotipológico distó de ser una práctica masiva, no se realizaron selecciones de migrantes, entre otros. En cambio, la eugenesia tuvo importante difusión e influencia en el Uruguay de 1900 a 1940 (Barrán, 1999: 49) y se concretó en diversos ámbitos.

En el ámbito médico la línea de acción fue la de evitar los matrimonios entre personas con enfermedades hereditarias, propuesta que surgió del Primer Congreso Médico Nacional en 1916, y que fue difundida y apoyada en notas periodísticas y ensayos, concretándose en 1927 con los certificados de salud para contraer matrimonio que otorgaba la Clínica Preventiva Municipal (Montevideo) y posteriormente los Consultorios Médicos Prenupciales del Ministerio de Salud Pública en todo el país (Barrán, 1999). En 1934, se aprobó el Código del Niño en el que se consagró la "principal medida eugenista de la legislación uruguaya" (Barrán, 1999: 47) que fue la de persuadir ${ }^{8}$ a los futuros cónyuges para que acudieran a los Consultorios Médicos Prenupciales del Ministerio de Salud Pública. Stepan (1991: 185) entiende que el Código del Niño de 1934 constituye una pieza paradigmática para entender la eugenesia latina; comienza con medidas de bienestar social, incluye ayudas para las familias numerosas, protección para niños abandonados, provisión de viviendas para los indigentes e instalación de los mencionados Consultorios Médicos Prenupciales. La autora apunta que la eugenesia latina incorporó medidas de protección, a diferencia de Estados Unidos que excluyó la preocupación por la salud y las medidas de bienestar social, y además que los certificados médicos prenupciales no fueron obligatorios sino sugeridos. Otra relevante aplicación de los postulados eugenistas en el Uruguay fue la despenalización del aborto en 1933 que permitió el aborto libre desde 1934 hasta que

8 Constituye una medida de coercitividad disimulada, concepto de Miranda y Vallejo que se presenta más adelante en este texto. 
fue derogado en 1938 (Sapriza, 2011). Dos leyes inmigratorias (1932 y 1936) contienen fundamentos eugenésicos que se evidencian en los discursos de justificación; la de 1932 - conocida como ley de indeseables - y sus reglamentaciones restringen el acceso de inmigrantes a Uruguay y la de 1936 agudiza las restricciones (Facal, 2002).

En el ámbito educativo, que es el que concierne a este artículo, la influencia fue intensa en la educación estatal en las décadas del veinte y treinta del siglo XX (Barrán, 1999: 50). Este autor señala dos hechos que evidencian la incorporación del eugenismo en la currícula: el texto de enseñanza "Higiene y Biología" de Francisco Brito del Pino, la existencia de la cátedra de Higiene Infantil y Puericultura del pediatra Américo Mola en la formación magisterial que instauró oficialmente el eugenismo en 1925 y un texto para docentes de 1932 .

En este marco, la cátedra de Higiene Social abordaba contenidos de eugenesia a partir de 1926. Dentro de su programa se incluía el punto "Las enfermedades hereditarias":

Las enfermedades hereditarias. La preservación de la raza. La Eugenesia. La Eugenética de Pinard. Educación y preparación de la juventud a sus deberes de progenitores. La necesidad de formar el sometimiento de la responsabilidad para con la descendencia. El desarrollo de la conciencia moral en materia de higiene profilaxis, tanto para con los demás como para con la especie, especialmente en lo que se relaciona con las enfermedades que repercuten sobre la descendencia. (Luisi, 1950a: 276)

De la lectura de los contenidos propuestos se desprende que su preocupación era la transmisión de la idea y sentimiento de responsabilidad para la descendencia. Insistía en la higiene y profilaxis de enfermedades que podían afectar a la descendencia, y por tanto a "la raza".

Para comprender la mirada eugenésica de Luisi conviene revisar tres de sus textos sobre este tema, los folletos "Algunas ideas sobre Eugenia" (Luisi, 1916), "Para una mejor descendencia" (Luisi, 1919) y apuntes manuscritos con el título "Tema 13. El eugenismo" que posiblemente fueran apuntes para el desarrollo de la cátedra.

Los folletos sobre eugenesia publicados por Luisi evidenciaban un elaborado discurso que fue llevado a la currícula de la cátedra de Higiene Social. Recientemente Rodríguez (2019) analizó la propuesta de educación sexual de Paulina Luisi y sus vínculos con el discurso eugenésico aunque sin considerar estos folletos, piezas clave para comprender la eugenesia de Luisi, por la descripción conceptual, el análisis de medidas eugenésicas que realizaba, la propuesta de un programa de medidas, los énfasis conceptuales y la preferencia por los postulados eugenésicos de Pinard.

Retomando a Armus, algunos estudios sobre eugenesia enfatizan en la existencia de una eugenesia positiva, medioambientalista y educativa propia del mundo latino, caracterizada por el lugar marginal de la esterilización y de violentos métodos de selección, frente a una eugenesia negativa en la que se promovió la esterilización, la castración e incluso la eutanasia de seres indeseables o que pudieran concebir hijos con enfermedades hereditarias, más propia del mundo anglosajón. Sin embargo, Paulina Luisi acordaba con la eugenesia positiva y con la negativa, como se verá.

Por su parte, el texto "Algunas ideas sobre Eugenia" mostraba el recorrido teórico que realizó estudiando algunas propuestas de "responsabilidad genésica", es decir, de cuidado sobre los nacimientos. Analizó allí los "procedimientos anticoncepcionales", la castración o esterilización eugenésica (que expresa que está en una fase experimental) y el aborto por causas eugenésicas. Acerca de las posibilidades de la utilización de 
procedimientos anticoncepcionales y de la esterilización eugenésica asumía que: "La no reproducción de los seres tarados física o mentalmente es, pues, una necesidad social (...) En nombre de la piedad para el individuo; en nombre del interés para la raza, nos permitimos afirmar que esos gérmenes deben ser destruidos" (Luisi, 1916: 18-19). Explicó su interés por la Eugenética de Pinard9 porque creía que el camino a recorrer era el de la determinación voluntaria de las condiciones de generación de la descendencia y esto se lograba con educación sexual.

Siguiendo esta lógica de razonamiento, Luisi sostuvo la idea de que hay personas que tienen "incapacidad" para engendrar ya que no se pueden dar las condiciones de "excelencia deseada para la procreación" (Luisi, 1916: 8). Presentó el pensamiento de diversos autores y numerosas medidas tomadas por los Estados en otras partes del mundo, ${ }^{10}$ sin embargo, para ella, las medidas estatales comentadas no eran suficientes, además de que esos procedimientos serían poco aplicables en el medio social uruguayo. Creía que había que sustituir el régimen penal por la "conciencia de la responsabilidad. Solamente la educación, el conocimiento de los factores benéficos y nocivos para las enfermedades de la raza, podrán educar esta conciencia y crear esta responsabilidad" (Luisi, 1916: 11).

Conceptualmente, según explicitó, confiaba más en la educación de la responsabilidad eugenésica pero, como venimos haciendo notar, en las conclusiones emite recomendaciones que incluyen medidas identificadas con la denominada "eugenesia negativa". Las recomendaciones para los Estados que Luisi realiza comprenden la necesidad de tomar "medidas sanitarias tendientes a proteger la reproducción de nuestra raza", la "acción profiláctica contra la degeneración de la especie", el dictado de "leyes severas" sobre la represión del alcoholismo y el consumo de sustancias tóxicas, "severas disposiciones sobre profilaxis venérea", "conveniencia social" de revisar la legislación sobre el aborto y que los Estados deberían tomar acción para "mejorar el medio de vida obrero" que empobrece o debilita el organismo de los futuros progenitores (Luisi, 1916: 25-26).

En el texto posterior publicado en 1919, "Para una mejor descendencia", se enfocó en la explicación de algunos conceptos eugenésicos y en la idea de evitar la procreación dominando al deseo sexual. Nótese que es el mismo momento en que estaba desarrollando las propuestas de educación sexual reseñadas al principio de este artículo.

Creía que, educando la conciencia de los adultos, podrían cambiar las condiciones de generación de otros seres. Se trataría así de educar en "cuestiones relativas a la perpetuación de la especie" (ibídem: 6).

9 Reggiani (2014) ubica a Adolphe Pinard como figura dominante de la eugenesia francesa hasta los años treinta, miembro fundador de la Sociedad Francesa de Eugenesia, pionero de la puericultura. En la "I Conferencia Internacional de Eugenesia" celebrada en Londres en 1912, Pinard definió la eugenesia como: "la puericultura antes de la procreación (...) tiene por objeto estudiar y dar a conocer las condiciones más favorables a la reproducción para aplicarlas de la mejor manera, con objeto de conservar y mejorar la especie humana" (Pinard en Luisi, 1916: 7).

10 Analiza, por ejemplo, la propuesta de Landouzy en Bruselas acerca de que en lugar de curar a los niños o niñas con tuberculosis se debería impedir su procreación por la profilaxis del matrimonio de personas con tuberculosis (y propone extender esta medida a todas las enfermedades que denomina degenerativas); la posibilidad de solicitar un "certificado de aptitud generadora" para contraer matrimonio como propuso Cazalis en Francia, Malthus que no se opone al matrimonio pero quiere prohibir la procreación, Mac Laren que propone la contratación de un seguro para garantizar la salud de los contrayentes de matrimonio, Schallmeyer que reclama impedir el matrimonio hasta un año después de la curación demostrada, Wylm que solicita examen médico para los futuros esposos y esposas, Zucarelli que pide la esterilización de criminales, Lombroso que apoya a este último. Revisa las realizaciones en Rumania en donde se discute la interdicción del matrimonio por causas de salud; en Dinamarca en donde el Código Penal llega a las relaciones conyugales al penar el contagio de enfermedad venérea de un cónyuge a otro; en algunos de los Estados de los EE.UU. Luisi anota que las medidas a tomar en los futuros matrimonios no protegen a los descendientes de uniones libres (Luisi, 1916: 8-11). 
La Eugénica en una de sus aplicaciones prácticas trata (...) de civilizar este instinto de la generación encaminándole a la producción de descendientes que señalen un progreso sobre sus antecesores. Este es también el concepto de la Eugenética de Pinard, o sea la "puericultura antes de la procreación", la que, como dice el viejo campeón de la defensa de la infancia "tiene por objeto estudiar y dar a conocer las condiciones más favorables a la reproducción para aplicarlas de la mejor manera, con objeto de conservar y mejorar la especie humana". Es, en suma, someter al instinto genésico a disposiciones o leyes, es, en otros términos, civilizarlo. (ibídem: 8)

La preocupación de Luisi radicaba en la prevención, en la puericultura antes de la procreación y es por esto que insistió en la mirada eugenésica de Pinard. De esto se infiere la importancia radical de la educación sexual para ella porque resultaba central para lograr una mejor descendencia y asegurar así el cumplimiento de las leyes de la evolución, particularmente de la selección natural.

Luisi desarrolló luego la manera en que se debe intervenir para "corregir" dificultades que enfrenta la selección natural en las sociedades humanas. Se trataría de determinar voluntariamente las condiciones en las que se efectúa la procreación de la siguiente manera:

Una ligera educación de los sentidos, una pequeña dosis de dominio sobre los deseos naturales, iluminados por un sencillo conocimiento de algunas causas que indefectiblemente repercuten sobre la generación, serían suficientes para evitar muchos males, muchos nacimientos de seres llamados a la vida en desastrosas condiciones fisiológicas que obran luego sobre toda la existencia. (Luisi, 1919: 8)

Su propuesta fue la de anteponer la "razón" al "deseo", el "dominio sobre los deseos naturales", es decir, el conocimiento razonado de las consecuencias del acto sexual llevaría a una represión del deseo sexual para evitar generar descendencia con enfermedades hereditarias. La oposición "reflexión/placer" quedaba clara también en el siguiente pasaje: "Si los esposos reflexionaran que el minuto de placer, irreflexivamente satisfecho, puede ser causa de la desgracia física o de la inferioridad fisiológica o psíquica de su descendencia, mirarían dos veces, a buen seguro, antes de darle el ser" (Luisi, 1919: 9). Según la autora, hay estados en los que no se debería engendrar; algunos son transitorios y otros permanentes: estado de fatiga, preocupación moral, exposición a tóxicos. Específicamente le preocupaban las "intoxicaciones crónicas" como la tuberculosis, la sífilis, las producidas por el alcohol, el tabaco, la morfina, la cocaína, el plomo u otros.

Sin embargo, a la tuberculosis, la sífilis y el alcoholismo les daba mayor peso, los llamaba el "triunvirato destructor de nuestra especie por dos mecanismos que se suman para agravar las consecuencias: disminución de la natalidad y degeneración de sus productos" (Luisi, 1919: 11). Pero no se queda allí en la enumeración de los estados en los que es preferible no engendrar porque describe otros estados que "aunque indirectamente, obran con fuerza indiscutible": falta de higiene, malas condiciones en los métodos de vida, hacinamiento, surmenage físico o mental, etc. (Luisi, 1919: 10). En este planteo se lee también una connotación de clase social: "Para la clase proletaria se agrava por insalubridad de las viviendas, deficiencia en la ración necesaria de aire y de luz; alimentación defectuosa o insuficiente" (Luisi, 1919: 11).

Ahora bien, una revisión de los conceptos de eugenesia positiva y negativa realizada por Marisa Miranda y Gustavo Vallejo (comentada por Armus, 2016) propone distinguir entre eugenesias de coercitividad explícita y de coercitividad disimulada. En ambos casos, se construye una "otredad" que es la que debe adaptarse. Leída desde esta perspectiva, la postura eugenésica de Luisi construye al otro desde concepciones médicas, morales y sociales y la coercitividad eugenésica para nada es disimulada. 
La eugenesia encontró lugar en la formación magisterial durante los años en que funcionó la cátedra. Las actividades adjuntas a la misma llevaron estas ideas a un público mayor. La síntesis que se encuentra en el manuscrito "Tema 13": el Eugenismo contiene algunas recomendaciones en relación con la higiene social, la educación, la legislación y la sociología. Este manuscrito no está fechado, pero presumiblemente fueran contenidos de la Cátedra de Higiene Social.

En este manuscrito, en relación con la higiene social, Luisi planteaba "que todo individuo portador de taras degenerativas susceptibles de ser heredadas o de dar productos degenerados o inferiores, debe ser privado temporaria o permanentemente del derecho natural de tener descendencia" (p. 1). La coercitividad eugenésica es explícita y se presenta este discurso en la currícula magisterial con concepciones de una otredad a corregir: la degeneración. ${ }^{11}$

Desde el punto de vista de la educación planteaba "que la enseñanza sexual y la profiláctica de todas las enfermedades sociales sea suministrada amplia y científicamente en todos los centros de educación y que ella comience desde la escuela elemental" (p. 1). Finalmente, desde el punto de vista de la legislación y la sociología proponía una completa emancipación sexual para la mujer, una sola moral para ambos sexos, el reconocimiento de la maternidad como una función del Estado y "que las organizaciones políticas y sociales y por lo tanto las leyes de todas ellas funden sus disposiciones civiles sobre la base del reconocimiento de la mujer como jefe directo de la familia; en otros términos, sobre la base del matriarcado, obedeciendo así al axioma derivado de los hechos naturales: los hijos pertenecen en primer término a la madre" (p. 2).

De este modo, Luisi hacía recaer la responsabilidad eugenésica en las mujeres, pero solicitaba, al mismo tiempo, el reconocimiento del Estado de la maternidad como función social.

\section{Líneas de cierre}

En este artículo se revisaron las propuestas y posicionamientos en torno a la educación sexual propuestos por Paulina Luisi, poniendo el foco en su experiencia de la cátedra de Higiene Social para estudiantes normalistas dictada entre 1926 y 1930. Tal como ha sugerido Darré (2008), su perfil intelectual, su dimensión política y sus polémicas, convirtieron a Luisi en un personaje muy popular, una figura emblemática en la historia uruguaya. En la historia de la educación sexual, particularmente, Luisi fue una pieza clave, ya que fue una de las primeras personalidades de su época en insistir con recurrencia en la importancia de esta instrucción, como un tema digno del debate público por sus consecuencias sociales y escolares.

Desde la cátedra, Luisi fue delineando y esbozando algunos tópicos y argumentos sobre el tipo de educación sexual que en su ideario debía hacerse extensivo en el sistema educativo público. Como se ha mencionado, según su mirada, este tipo de instrucción debía iniciarse con la enseñanza de las enfermedades venéreas dentro de un conjunto de enfermedades sociales y su profilaxis. Sin embargo, Luisi creía que la enseñanza sexual no se agotaba en la prevención del contagio de enfermedades, sino que debía articularse con el estudio y fundamentos de la Eugenesia, insistiendo en la necesidad de fomentar la idea y el sentimiento de "responsabilidad para con la descendencia". A esto se agregaban los deberes en materia sexual para con uno mismo, para con el otro sexo y para con la descendencia. Así, de la moral biológica se deducía una moral práctica.

11 Sobre el significado de “degeneración” para los eugenistas, ver Reggiani (2014). 
El objeto de la enseñanza sexual, contenido en los planteos de Luisi, era la castidad en ambos sexos hasta la vida adulta, el sometimiento del instinto sexual a la acción de la voluntad, el control de la seducción y del deseo, la condena de las lecturas que incitaban al deseo, la frecuentación de cabarets y el uso de pornografía. Al mismo tiempo, como lo hiciera el feminismo de su época, condenó la doble moral para los sexos en materia sexual, defendiendo una moral única para varones y mujeres en cuanto a la corresponsabilidad en las consecuencias del acto sexual.

En su discurso pedagógico, institucionalizado en la cátedra en la ciudad de Montevideo, resultaba clave preparar a estudiantes normalistas porque creía que la enseñanza sexual debía realizarse en todos los ámbitos educativos, comenzando por la escuela primaria. En sus propuestas hacia el sistema educativo - que no se consolidaron, salvo para estudiantes normalistas-, Luisi propuso a los maestros como actores relevantes en la educación sexual, de allí que la preparación de los mismos en los cursos sobre educación sexual no debía ser solamente de carácter teórico, sino también debía incorporar tareas prácticas en escuelas.

Analizamos también que los planteos eugenésicos constituyeron esencialmente gran parte de la fundamentación de su propuesta de educación sexual. El dominio sobre el instinto o los deseos sexuales sería posible a partir de la enseñanza de conocimientos sobre herencia y enfermedades hereditarias que permitieran la "responsabilidad genésica". De allí que incluyera contenidos sobre eugenesia coercitiva en su cátedra; por tanto, en su planteo, la finalidad de la educación sexual, era mejorar las condiciones de la reproducción humana. Como se mostró, en pro de este mejoramiento de la raza propuso el "control de los instintos" y la "represión de los deseos" que se tradujo en la abstinencia ante el acto sexual a fin de reservarse para los momentos en que se estuviera en condiciones de "excelencia" para la procreación. Así, Luisi significó también un capítulo importante para la tradición del eugenismo: en nombre de la eugenesia se dictan severas formas de vivenciar la sexualidad atada a las finalidades de la reproducción biológica, siendo la educación de la sexualidad una estrategia privilegiada para ese fin. 


\section{Q Referencias bibliográficas}

"Armus, D. (2016). Eugenesia en Buenos Aires: discursos, prácticas, historiografía. História, Ciências, Saúde, vol. 23, supl., dez.: 149-169. Río de Janeiro, Manguinhos.

" Barrán, J. P. (1992). Medicina y sociedad en el Uruguay del Novecientos, 1. El poder de curar, 1ª reimpr. Montevideo, Ediciones de la Banda Oriental.

» --------. (1995). Medicina y sociedad en el Uruguay del Novecientos, 3. La invención del cuerpo. Montevideo, Ediciones de la Banda Oriental.

» --------. (1999). Biología, Medicina y eugenesia en Uruguay. Asclepio, Vol. LI (2): 11-50.

» Biernat, C. (2007). Médicos, especialistas, políticos y funcionarios en la Organización centralizada de la profilaxis de las enfermedades venéreas en la Argentina (1930-1954). Anuario de Estudios Americanos, 64, 1, enero-junio: 257288. Sevilla.

"Darré, S. (2005). Políticas de género y discurso pedagógico. La educación sexual en el Uruguay del siglo XX. Montevideo, Trilce.

»-------. (2008). Cuando el saber no tiene lugar: la difícil implementación de la educación sexual en el sistema educativo uruguayo. En Araújo. K. y Prieto, M. Estudios sobre sexualidades en América Latina, pp. 199-213. Quito, FLACSO Ecuador.

"Díaz Berenguer, A. (2012). La Medicina del Novecientos: Poder y Saber. Archivos de Medicina Interna, 34(3), 95-101. Disponible en: http://www.scielo.edu.uy/ scielo.php? script=sci_arttext\&pid=S1688-423X2012000300006\&lng=es\&tlng= es (Consultado el 24/07/2020).

"Di Liscia, M. S. (2008). Los bordes y límites de la eugenesia, donde caen las "razas superiores" (Argentina, primera mitad del siglo XX). En Miranda, M. y Vallejo G. Políticas del cuerpo. Estrategias modernas de normalización del individuo y la sociedad, pp. 377-409. Buenos Aires, Siglo XXI.

»Espiga, S. (2015a). Paulina Luisi: de la instrucción sexual a la educación sexual Historia y docencia. APHU, Año VI, N 5, dic.: 6-18.

"--------. (2015b). La educación sexual para los niños. En Espiga, S. La infancia normalizada. Libros, maestros e higienistas en la escuela pública uruguaya 18851918, pp. 149.16o. Montevideo, Antítesis.

»Facal, S. (2002). Política inmigratoria de puertas cerradas. Uruguay frente a la llegada de refugiados españoles republicanos y judíos alemanes. Revista Complutense de Historia de América, Vol. 28: 169-183.

"Foucault, M. (1998). Historia de la sexualidad 1: la voluntad de saber. México, Siglo Veintiuno.

" Giordano, V. (2012). Ciudadanas incapaces. La construcción de los derechos civiles de las mujeres en Argentina, Brasil, Chile y Uruguay en el siglo XX. Buenos Aires, Teseo.

» Jones, D.; Figari, C. y Barrón López, S. (2012). La producción de la sexualidad. Políticas y regulaciones sexuales en Argentina. Buenos Aires, Biblos.

"Lavrin, A. (2005). Mujeres, feminismo y cambio social en Argentina, Chile y Uruguay 1890-1940. Santiago de Chile, Ediciones de la Dirección de Bibliotecas, Archivos y Museos. 
"Miranda, M. (2005). Matrimonio y procreación en la ortodoxia eugénica argentina. Cuadernos del CISH, $\mathrm{N}^{\circ}$ 17-18: 1-28.

$»$--------. (2011). Controlar lo incontrolable. Una historia de la sexualidad argentina. Buenos Aires, Biblos.

»Miranda, M. y Vallejo, G. (comps.). (2005). Darwinismo social y eugenesia en el mundo latino. Buenos Aires, Siglo XXI.

»------. (2014). Iglesia, eugenesia y control de la moral sexual: apuntes para una historia del onanismo (1930-1970). En Barrancos, D.; Guy, D. y Valobra, A. Moralidades y comportamientos sexuales. Argentina, 1880-2011. Buenos Aires, Biblos.

"Nari, M. (2004). Políticas de maternidad y maternalismo político. Buenos Aires (1890-1920). Buenos Aires, Biblos.

» Palma, H. A. y Palma, D. (2007). Eugenesia y ética. En Miranda, M. y Vallejo G. Políticas del cuerpo. Estrategias modernas de normalización del individuo y la sociedad, pp. 521-542. Buenos Aires, Siglo XXI.

"Palomeque, A. (2012). Historia de la educación uruguaya. Tomo 3: La educación uruguaya 1886-1930. Montevideo, Ediciones de la Plaza.

»Pecheny, M. (2009). Parece que no fue ayer: el legado de la Ley de Divorcio en perspectiva de derechos sexuales. En Gargarella, R.; Murillo, M. V. y Pecheny, M. Discutir Alfonsín, Buenos Aires, Siglo XXI.

»Queirolo, G. (2013). Género y sexualidad en tiempos de males venéreos (Buenos Aires, 1920-1940). Revista Nomadías, № 17, jul.: 67-87.

»Reggiani, A. (2014). Eugenesia y cultura física. Tres trayectorias históricas: Francia, Gran Bretaña, Argentina. En Scharagrodsky, P. (comp.). Miradas médicas sobre la cultura física. Argentina 1880-1970, pp. 17-58. Buenos Aires, Prometeo.

"Rodríguez, C. (2019). A cien años del primer plan y métodos de enseñanza sexual en el Uruguay. Sus vinculaciones con el discurso eugenésico. Anuario de Historia de la Educación (SAHE), Vol. 20, № 2: 75-89.

"Sapriza, G. (1988). Paulina Luisi. En Sapriza, G. Memorias de rebeldía. Siete historias de vida, pp. 77-107. Montevideo, Puntosur.

»-------. (2008 [2006]). La hora de la eugenesia: las feministas en la encrucijada. En Morant, I. (dir.). Historia de las mujeres en España y América Latina III del siglo XIX a los umbrales del XX, 2ª ed., pp. 889-913. Madrid, Cátedra.

»-------. (2011). Historia de la (des) penalización del aborto en Uruguay. “Aborto libre": la corta experiencia uruguaya (1934-1938). En AA.VV. (Des) penalización del aborto en Uruguay: prácticas, actores y discursos, pp. 19-64. Montevideo, Universidad de la República-CSIC.

"Scharagrodsky, P.; Zemaitis, S. (2020). De vicios y extrañas perversiones durante la adolescencia. Sexualidades, sexos y relaciones de género en la paidología de Víctor Mercante (Argentina, principios del siglo XX). En Baquero, R.; Scharagrodsky P. y Porro, S. Discursos, prácticas e instituciones educativas, pp. 105-132. Buenos Aires, Prometeo.

» Sosa, F. (2018). Acceso de mujeres a la educación secundaria pública en el Uruguay (1911-1936): femineidades, sexualidades y maternidades (Tesis de Maestría). Buenos Aires: FLACSO Sede Académica Argentina.

» Southwell, M. (2011). Lo social como interpelación pedagógica: mujeres en disputa con sus épocas. En Krichesky, M. Pedagogía social y educación popular. Perspectivas y estudios sobre la inclusión y el derecho a la educación. La Plata, UNIPE. 
"Stepan, N. (1991). The hour of Eugenics. Race, Gender and Nation in Latin America. Ithaca/Londres, Cornell University Press.

"Zemaitis, S. (2016). Pedagogías de la sexualidad. Antecedentes, conceptos e historia en el campo de la educación sexual de la juventud. (Trabajo Final Integrador. Especialización en Nuevas Infancias y Juventudes). La Plata, Universidad Nacional de La Plata- Facultad de Humanidades y Ciencias de la Educación. Disponible en: http://sedici.unlp.edu.ar/handle/10915/52421 (Consultado el 23/12/2020)

\section{Fuentes citadas}

"Camaña, R. (1910). El prejuicio del profesorado en la Facultad de Filosofía y Letras. Derecho, Historia y Letras. Tomo 37.

»--------. (1912). La educación sexual. Buenos Aires, Talleres Gráficos Riachuelo.

» --------. (1916). Pedagogía Social. Buenos Aires, La Cultura Argentina.

» “La doctora Paulina Luisi. Datos biográficos”, 12/1925, Acción Femenina: 2-10.

"Consejo Nacional de Higiene (1906). Profilaxia de las enfermedades venéreosifilíticas. Montevideo, El Siglo llustrado.

» Luisi, P. (1916). Algunas ideas sobre Eugenia. Montevideo, El Siglo llustrado.

"-------. (1919). Para una mejor descendencia. Buenos Aires, Casa Editora Juan Perrotti. (folleto).

»------. (1922). “Plan y métodos de Enseñanza Sexual”. En Anales de Instrucción Primaria, Tomo XIX, $\mathrm{n}^{\circ} 1,2$ y 3, enero, febrero y marzo:, 197-223. Montevideo, Barreiro y Ramos.

»------. (1925). Curso de Higiene Social. Conferencia Qué es la Higiene Social, 10 septiembre 1925-13 mayo 1926, manuscrito en carpeta Higiene Social en Archivo Paulina Luisi del Archivo Literario de Biblioteca Nacional del Uruguay.

»--------. (1944). Manuscrito en carpeta Educación Sexual 1, en Archivo Paulina Luisi del Archivo Literario de Biblioteca Nacional del Uruguay.

》--------. (1950a). Una Cátedra de Higiene Social. En Pedagogía y conducta sexual, pp. 247-315. Montevideo, El Siglo Ilustrado.

»-------. (1950b). Ensayo sobre Enseñanza biológico-eugenésica (DoctrinaMetodología). En Pedagogía y conducta sexual, pp. 3-19. Montevideo, El Siglo llustrado.

»-------. (s/d). Tema № 13. El Eugenismo, manuscrito en Archivo Paulina Luisi del Archivo Literario de Biblioteca Nacional del Uruguay.

"Rodríguez, S. B. (1909). Cartilla sobre la Tuberculosis y Nociones de Higiene Preventiva escrita expresamente para uso de las escuelas y del Pueblo en general. Montevideo, El Siglo llustrado. 
